\title{
Efficient Type Matching
}

\author{
Somesh Jha ${ }^{1}$, Jens Palsberg ${ }^{2}$, and Tian Zhao ${ }^{2}$ \\ 1 Computer Sciences Department, \\ University of Wisconsin, Madison, WI 53706, \\ jha@cs.wisc.edu. \\ 2 Department of Computer Science, \\ Purdue University, W. Lafayette, IN 47907, \\ \{palsberg,tzhao\}@cs.purdue.edu.
}

\begin{abstract}
Palsberg and Zhao [17] presented an $O\left(n^{2}\right)$ time algorithm for matching two recursive types. In this paper, we present an $O(n \log n)$ algorithm for the same problem. Our algorithm works by reducing the type matching problem to the well-understood problem of finding a size-stable partition of a graph. Our result may help improve systems, such as Polyspin and Mockingbird, that are designed to facilitate interoperability of software components. We also discuss possible applications of our algorithm to Java. Issues related to subtyping of recursive types are also discussed.
\end{abstract}

\section{Introduction}

Interoperability is a fundamental problem in software engineering. Interoperability issues arise in various contexts, such as software reuse, distributed programming, use of legacy components, and integration of software components developed by different organizations. Interoperability of software components has to address two fundamental problems: matching and bridging. Matching deals with determining whether two components $A$ and $B$ are compatible, and bridging allows one to use component $B$ using the interface defined for component $A$.

Matching: A common technique for facilitating matching is to associate signatures with components. These signatures can then be used as keys to retrieve relevant components from an existing library of components. Use of finite types as signatures was first proposed by Rittri [19]. Zaremski and Wing [23,24] used a similar approach for retrieving components from an ML-like functional library. Moreover, they also emphasized flexibility and support for user-defined types.

Bridging: In a multi-lingual context, bridge code for "gluing" components written in different languages (such as $\mathrm{C}, \mathrm{C}++$, and Java) has to be developed. CORBA [15], PolySpin [4], and Mockingbird [23] allow composing components implemented in different languages. Software components are considered to be of two kinds: objects, which provide public interfaces, and clients, which invoke the methods of the objects and thereby use the services provided by the objects.

\footnotetext{
* Palsberg was supported by an NSF CAREER award, CCR-9734265, and by IBM. 
The Problem: Assume that we use types as signatures for components. Thus, the type matching problem reduces to the problem of determining whether two types are equivalent. Much previous work on type matching focuses on non-recursive types [7 10,14 18], $[192021,23]$. In this paper, we consider equivalence of recursive types. Equality and subtyping of recursive types have been studied in the 1990s by Amadio and Cardelli [1]; Kozen, Palsberg, and Schwartzbach [13]; Brandt and Henglein [6]; Jim and Palsberg [12]; and others. These papers concentrate on the case where two types are considered equal if their infinite unfoldings are identical. In this case, type equivalence can be decided in $O(n \alpha(n))$ time. If we allow a product-type constructor to be associative and commutative, then two recursive types may be considered equal without their infinite unfoldings being identical. Alternatively, think of a product type as a multiset, by which associativity and commutativity are obtained for free. Such flexibility has been advocated by Auerbach, Barton, and Raghavachari [2]. Palsberg and Zhao [17] presented a definition of type equivalence that supports this idea. They also presented an $O\left(n^{2}\right)$ time algorithm for deciding their notion of type equivalence.

A notion of subtyping defined by Amadio and Cardelli [1] can be decided in $O\left(n^{2}\right)$ time [13]. We also briefly discuss subtyping of recursive types in this paper.

Our result: We present an $O(n \log n)$ time algorithm for deciding the type equivalence of Palsberg and Zhao [17]. Our algorithm works by reducing the type matching problem to the well-understood problem of finding a size-stable partition of a graph [16].

The organization of the paper: A small example is described in Section 2. This example will be used throughout the paper for illustrative purposes. In Section 3 we recall the notions of terms and term automata [18,11 13], and we state the definitions of types and type equivalence from the paper by Palsberg and Zhao [17]. In Section 4 we prove our main result. An implementation of our algorithm is discussed Section 5 , Subtyping of recursive types is discussed in Section 6. Concluding remarks appear in Section 7

\section{Example}

In this section we provide a small example which will be used throughout the paper. It is straightforward to map a Java type to a recursive type of the form considered in this paper. A collection of method signatures can be mapped to a product type, a single method signature can be mapped to a function type, and in case a method has more than one argument, the list of arguments can be mapped to a product type. Recursion, direct or indirect, is expressed with the $\mu$ operator. This section provides an example of of Java interfaces and provides an illustration of our algorithm.

Suppose we are given the two sets of Java interfaces shown in Figures 1 and 2, We would like to find out whether interface $I_{1}$ is "structurally equal" to or "matches" with interface $J_{2}$. We want a notion of equality for which interface names and method names do not matter, and for which the order of the methods in an interface and the order of the arguments of a method do not matter.

Notice that interface $I_{1}$ is recursively defined. The method $m_{1}$ takes an argument of type $I_{1}$ and returns a floating point number. In the following, we use names of interfaces and methods to stand for their type structures. The type of method $m_{1}$ can be expressed 


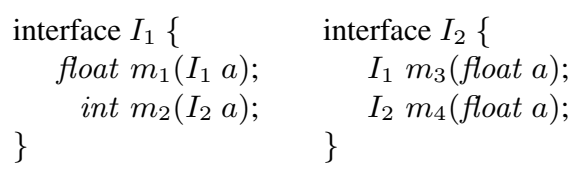

Fig. 1. Interfaces $I_{1}$ and $I_{2}$

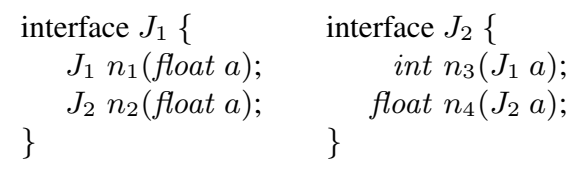

Fig. 2. Interfaces $J_{1}$ and $J_{2}$

as $I_{1} \rightarrow$ float. The symbol $\rightarrow$ stands for the function type constructor. Similarly, the type of $m_{2}$ is $I_{2} \rightarrow$ int. We can then capture the structure of $I_{1}$ with conventional $\mu$-notation for recursive types:

$$
I_{1}=\mu \alpha .(\alpha \rightarrow \text { float }) \times\left(I_{2} \rightarrow \text { int }\right)
$$

The symbol $\alpha$ is the type variable bound to the type $I_{1}$ by the symbol $\mu$. The interface type $I_{1}$ is a product type with the symbol $\times$ as the type constructor. Since we think of the methods of interface $I_{1}$ as unordered, we could also write the structure of $I_{1}$ as

$$
\begin{aligned}
& I_{1}=\mu \alpha .\left(I_{2} \rightarrow \text { int }\right) \times(\alpha \rightarrow \text { float }), \\
& I_{2}=\mu \delta .\left(\text { float } \rightarrow I_{1}\right) \times(\text { float } \rightarrow \delta) .
\end{aligned}
$$

In the same way, the structures of the interfaces $J_{1}, J_{2}$ are:

$$
\begin{aligned}
& J_{1}=\mu \beta .(\text { float } \rightarrow \beta) \times\left(\text { float } \rightarrow J_{2}\right) \\
& J_{2}=\mu \eta .\left(J_{1} \rightarrow \text { int }\right) \times(\eta \rightarrow \text { float }) .
\end{aligned}
$$

Trees corresponding to the two types are shown in Figures 3 and 4 The interface types $I_{1}, J_{2}$ are equivalent iff there exists a one-to-one mapping or a bijection from the methods in $I_{1}$ to the methods in $J_{2}$ such that each pair of methods in the bijection relation have the same type. The types of two methods are equal iff the types of the arguments and the return types are equal.
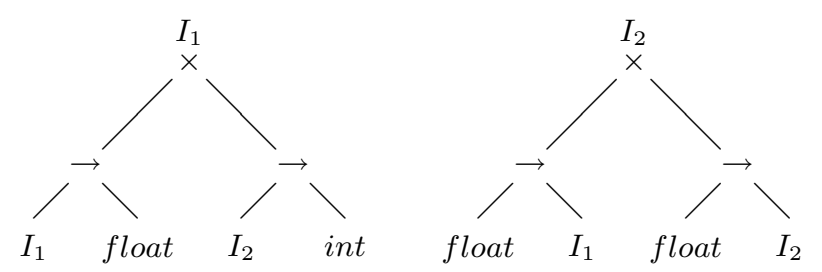

Fig. 3. Trees for interfaces $I_{1}$ and $I_{2}$ 

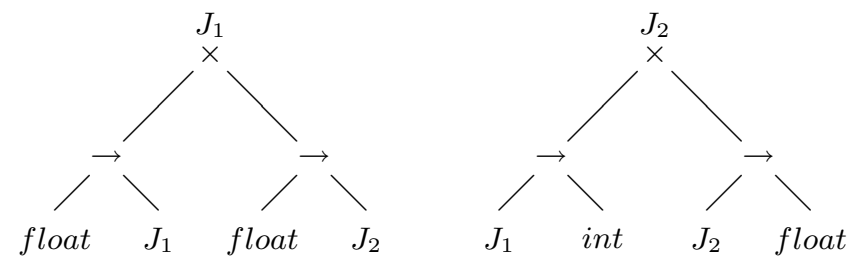

Fig. 4. Trees for interfaces $J_{1}$ and $J_{2}$

The equality of the interface types $I_{1}$ and $J_{2}$ can be determined by trying out all possible orderings of the methods in each interface and comparing the two types in the form of finite automata. In this case, there are only few possible orderings. However, if the number of methods is large and/or some methods take many arguments, the above approach becomes time consuming because the number of possible orderings grows exponentially. An efficient algorithm for determining equality of recursive types will be given later in the paper.

\section{Definitions}

A recursive type is a type described by a set of equations involving the $\mu$ operator. An example of a recursive type was provided in Section 2. This section provides representation of recursive types as terms and term automata.

Term automata and representation of types are described in Subsection 3.1. A definition of type equivalence for recursive types in terms of bisimulation is give in Subsection 3.2 An efficient algorithm for determining whether two types are equivalent is given in Section 4

\subsection{Terms and Term Automata}

Here we give a general definition of (possibly infinite) terms over an arbitrary finite ranked alphabet $\Sigma$. Such terms are essentially labeled trees, which we represent as partial functions labeling strings over $\omega$ (the natural numbers) with elements of $\Sigma$.

Let $\Sigma_{n}$ denote the set of elements of $\Sigma$ of arity $n$. Let $\omega$ denote the set of natural numbers and let $\omega^{*}$ denote the set of finite-length strings over the alphabet $\omega$.

A term over $\Sigma$ is a partial function

$$
t: \omega^{*} \rightarrow \Sigma
$$

satisfying the following properties:

- the domain of $t$ is nonempty and prefix-closed;

- if $t(\alpha) \in \Sigma_{n}$, then $\{i \mid \alpha i \in$ the domain of $t\}=\{0,1, \ldots, n-1\}$.

Let $t$ be a term and $\alpha \in \omega^{*}$. Define the partial function $t \downarrow \alpha: \omega^{*} \rightarrow \Sigma$ by $t \downarrow \alpha(\beta)=$ $t(\alpha \beta)$. If $t \downarrow \alpha$ has nonempty domain, then it is a term, and is called the subterm of t at position $\alpha$. 
A term $t$ is said to be regular if it has only finitely many distinct subterms; that is, if $\left\{t \downarrow \alpha \mid \alpha \in \omega^{*}\right\}$ is a finite set.

Every regular term over a finite ranked alphabet $\Sigma$ has a finite representation in terms of a special type of automaton called a term automaton. A term automaton over $\Sigma$ is a tuple

$$
A=\left(Q, \Sigma, q_{0}, \delta, \ell\right)
$$

where:

- $Q$ is a finite set of states,

- $q_{0} \in Q$ is the start state,

- $\delta: Q \times \omega \rightarrow Q$ is a partial function called the transition function, and

- $\ell: Q \rightarrow \Sigma$ is a (total) labeling function,

such that for any state $q \in Q$, if $\ell(q) \in \Sigma_{n}$ then

$$
\{i \mid \delta(q, i) \text { is defined }\}=\{0,1, \ldots, n-1\} .
$$

The partial function $\delta$ extends naturally to an inductively-defined partial function:

$$
\begin{aligned}
\hat{\delta} & : Q \times \omega^{*} \rightarrow Q \\
\hat{\delta}(q, \epsilon) & =q \\
\hat{\delta}(q, \alpha i) & =\delta(\hat{\delta}(q, \alpha), i) .
\end{aligned}
$$

For any $q \in Q$, the domain of the partial function $\lambda \alpha \cdot \hat{\delta}(q, \alpha)$ is nonempty (it always contains $\epsilon$ ) and prefix-closed. Moreover, because of the condition on the existence of $i$-successors in the definition of term automata, the partial function

$$
\lambda \alpha \cdot \ell(\hat{\delta}(q, \alpha))
$$

is a term.

Let $A$ be a term automaton. The term represented by $A$ is the term

$$
t_{A}=\lambda \alpha \cdot \ell\left(\hat{\delta}\left(q_{0}, \alpha\right)\right) .
$$

A term $t$ is said to be representable if $t=t_{A}$ for some $A$.

Intuitively, $t_{A}(\alpha)$ is determined by starting in the start state $q_{0}$ and scanning the input $\alpha$, following transitions of $A$ as far as possible. If it is not possible to scan all of $\alpha$ because some $i$-transition along the way does not exist, then $t_{A}(\alpha)$ is undefined. If on the other hand $A$ scans the entire input $\alpha$ and ends up in state $q$, then $t_{A}(\alpha)=\ell(q)$.

It is straightforward to show that a term $t$ is regular if and only if it is representable. Moreover, a term $t$ is regular if and only if it can be described by a finite set of equations involving the $\mu$ operator.

\subsection{Equivalence of Recursive Types}

A recursive type is a regular term over the ranked alphabet

$$
\Sigma=\Gamma \cup\{\rightarrow\} \cup\left\{\prod^{n}, n \geq 2\right\}
$$


where $\Gamma$ is a set of base types, $\rightarrow$ is binary, and $\prod^{n}$ is of arity $n$. Given a type $\sigma$, if $\sigma(\epsilon)=\rightarrow, \sigma(0)=\sigma_{1}$, and $\sigma(1)=\sigma_{2}$, then we write the type as $\sigma_{1} \rightarrow \sigma_{2}$. If $\sigma(\epsilon)=\prod^{n}$ and $\sigma(i)=\sigma_{i}, \forall i \in\{0,1, \ldots, n-1\}$, then we write the type $\sigma$ as $\prod_{i=0}^{n-1} \sigma_{i}$.

Palsberg and Zhao [17] presented three equivalent definitions of type equivalence. Here we will work with the one which is based on the idea of bisimilarity. A relation $R$ on types is called a bisimulation if it satisfies the following three conditions:

- if $(\sigma, \tau) \in R$, then $\sigma(\epsilon)=\tau(\epsilon)$

- if $\left(\sigma_{1} \rightarrow \sigma_{2}, \tau_{1} \rightarrow \tau_{2}\right) \in R$, then $\left(\sigma_{1}, \tau_{1}\right) \in R$ and $\left(\sigma_{2}, \tau_{2}\right) \in R$

- if $\left(\prod_{i=0}^{n-1} \sigma_{i}, \prod_{i=0}^{n-1} \tau_{i}\right) \in R$, then there exists a bijection $b:\{0 . . n-1\} \rightarrow\{0 . . n-1\}$ such that $\forall i \in\{0 . . n-1\},\left(\sigma_{i}, \tau_{b(i)}\right) \in R$.

Bisimulations are closed under union, therefore, there exists a largest bisimulation

$$
\mathcal{R}=\bigcup\{R \mid R \text { is a bisimulation }\} .
$$

It is straightforward to show that $\mathcal{R}$ is an equivalence relation. Two types $\tau_{1}$ and $\tau_{2}$ are said to be equivalent (denoted by $\tau_{1} \cong \tau_{2}$ ) iff $\left(\tau_{1}, \tau_{2}\right) \in \mathcal{R}$.

\section{An Efficient Algorithm for Type Equivalence}

Assume that we are given two types $\tau_{1}$ and $\tau_{2}$ that are represented as two term automata $A_{1}$ and $A_{2}$. Lemma 1 proves that $\tau_{1} \cong \tau_{2}$ (or $\left(\tau_{1}, \tau_{2}\right) \in \mathcal{R}$ ) if and only if there is a reflexive bisimulation $C$ between $A_{1}$ and $A_{2}$ such that the initial states of the term automata $A_{1}$ and $A_{2}$ are related by $C$. Lemma 3 essentially reduces the problem of finding a reflexive bisimulation $C$ between $A_{1}$ and $A_{2}$ to finding a size-stable coarsest partition [16]. Theorem[1] uses the algorithm of Paige and Tarjan to determine in $O(n \log n)$ time ( $n$ is the sum of the sizes of the two term automata) whether there exists a reflexive bisimulation $C$ between $A_{1}$ and $A_{2}$.

Throughout this section, we will use $A_{1}, A_{2}$ to denote two term automata over the alphabet $\Sigma$ :

$$
\begin{aligned}
& A_{1}=\left(Q_{1}, \Sigma, q_{01}, \delta_{1}, \ell_{1}\right) \\
& A_{2}=\left(Q_{2}, \Sigma, q_{02}, \delta_{2}, \ell_{2}\right) .
\end{aligned}
$$

We assume that $Q_{1} \cap Q_{2}=\emptyset$. Define $Q=Q_{1} \cup Q_{2}, \delta: Q \times \omega \rightarrow Q$ where $\delta=\delta_{1} \oplus \delta_{2}$, and $\ell: Q \rightarrow \Sigma$, where $\ell=\ell_{1} \oplus \ell_{2}$, where $\oplus$ denotes disjoint union of two functions. We say that $A_{1}, A_{2}$ are bisimular if and only if there exists a relation $C \subseteq Q \times Q$, called a bisimulation between $A_{1}$ and $A_{2}$, such that:

- if $\left(q, q^{\prime}\right) \in C$, then $\ell(q)=\ell\left(q^{\prime}\right)$

- if $\left(q, q^{\prime}\right) \in C$ and $\ell(q)=\rightarrow$, then $\left(\delta(q, 0), \delta\left(q^{\prime}, 0\right)\right) \in C$ and $\left(\delta(q, 1), \delta\left(q^{\prime}, 1\right)\right) \in C$

- if $\left(q, q^{\prime}\right) \in C$ and $\ell(q)=\prod^{n}$, then there exists a bijection $b:\{0 . . n-1\} \rightarrow$ $\{0 . . n-1\}$ such that $\forall i \in\{0 . . n-1\}:\left(\delta(q, i), \delta\left(q^{\prime}, b(i)\right)\right) \in C$.

Notice that the bisimulations between $A_{1}$ and $A_{2}$ are closed under union, therefore, there exists a largest bisimulation between $A_{1}$ and $A_{2}$. It is straightforward to show that the identity relation on $Q$ is a bisimulation, and that any reflexive bisimulation is an equivalence relation. Hence, the largest bisimulation is an equivalence relation. 
Lemma 1. For types $\tau_{1}, \tau_{2}$ that are represented by the term automata $A_{1}, A_{2}$, respectively, we have $\left(\tau_{1}, \tau_{2}\right) \in \mathcal{R}$ if and only if there is a reflexive bisimulation $C$ between $A_{1}$ and $A_{2}$ such that $\left(q_{01}, q_{02}\right) \in C$.

Proof. Suppose $\left(\tau_{1}, \tau_{2}\right) \in \mathcal{R}$. Define:

$$
C=\left\{\left(q, q^{\prime}\right) \in Q \times Q \mid\left(\lambda \alpha \cdot \ell(\hat{\delta}(q, \alpha)), \lambda \alpha \cdot \ell\left(\hat{\delta}\left(q^{\prime}, \alpha\right)\right)\right) \in \mathcal{R}\right\} .
$$

It is straightforward to show that $C$ is a bisimulation between $A_{1}$ and $A_{2}$, and that $\left(q_{01}, q_{02}\right) \in C$, we omit the details.

Conversely, let $C$ be a reflexive bisimulation between $A_{1}$ and $A_{2}$ such that $\left(q_{01}, q_{02}\right) \in$ $C$. Define:

$$
R=\left\{\left(\sigma_{1}, \sigma_{2}\right) \mid\left(q, q^{\prime}\right) \in C \wedge \sigma_{1}=\lambda \alpha \cdot \ell(\hat{\delta}(q, \alpha)) \wedge \sigma_{2}=\lambda \alpha \cdot \ell\left(\hat{\delta}\left(q^{\prime}, \alpha\right)\right)\right\}
$$

From $\left(q_{01}, q_{02}\right) \in C$, we have $\left(\tau_{1}, \tau_{2}\right) \in R$. It is straightforward to prove that $R$ is a bisimulation, we omit the details. From $\left(\tau_{1}, \tau_{2}\right) \in R$ and $R$ being a bisimulation, we conclude that $\left(\tau_{1}, \tau_{2}\right) \in \mathcal{R}$.

A partitioned graph is a 3-tuple $(U, E, P)$, where $U$ is a set of nodes, $E \subseteq U \times U$ is an edge relation, and $P$ is a partition of $U$. A partition $P$ of $U$ is a set of pairwise disjoint subsets of $U$ whose union is all of $U$. The elements of $P$ are called its blocks. If $P$ and $S$ are partitions of $U$, then $S$ is a refinement of $P$ if and only if every block of $S$ is contained in a block of $P$.

A partition $S$ of a set $U$ can be characterized by an equivalence relation $K$ on $U$ such that each block of $S$ is an equivalence class of $K$. If $U$ is a set and $K$ is an equivalence relation on $U$, then we use $U / K$ to denote the partition of $U$ into equivalence classes for $K$.

A partition $S$ is size-stable with respect to $E$ if and only if for all blocks $B_{1}, B_{2} \in S$, and for all $x, y \in B_{1}$, we have $\left|E(x) \cap B_{2}\right|=\left|E(y) \cap B_{2}\right|$, where $E(x)$ is the following set

$$
\{y \mid(x, y) \in E\} .
$$

If $E$ is clear from the context, we will simply use size-stable. We will repeatedly use the following characterization of size-stable partitions.

Lemma 2. For an equivalence relation $K$, we have that $U / K$ is size-stable if and only if for all $\left(u, u^{\prime}\right) \in K$, there exists a bijection $\pi: E(u) \rightarrow E\left(u^{\prime}\right)$ such that for all $u_{1} \in E(u)$, we have $\left(u_{1}, \pi\left(u_{1}\right)\right) \in K$.

Proof. Suppose that $U / K$ is size-stable. Let $\left(u, u^{\prime}\right) \in K$. Let $B_{1}$ be the block of $U / K$ which contains $u$ and $u^{\prime}$. For each block $B_{2}$ of $U / K$, we have that $\left|E(u) \cap B_{2}\right|=$ $\left|E\left(u^{\prime}\right) \cap B_{2}\right|$. So, for each block $B_{2}$ of $U / K$, we can construct a bijection from $E(u) \cap B_{2}$ to $E\left(u^{\prime}\right) \cap B_{2}$, such that for all $u_{1} \in E(u) \cap B_{2}$, we have $\left(u_{1}, \pi\left(u_{1}\right)\right) \in K$. These bijections can then be merged to single bijection $\pi: E(u) \rightarrow E\left(u^{\prime}\right)$ with the desired property.

Conversely, suppose that for all $\left(u, u^{\prime}\right) \in K$, there exists a bijection $\pi: E(u) \rightarrow$ $E\left(u^{\prime}\right)$ such that for all $u_{1} \in E(u)$, we have $\left(u_{1}, \pi\left(u_{1}\right)\right) \in K$. Let $B_{1}, B_{2} \in U / K$, and 
let $x, y \in B_{1}$. We have that $(x, y) \in K$, so there exists a bijection $\pi: E(x) \rightarrow E(y)$ such that for all $u_{1} \in E(x)$, we have $\left(u_{1}, \pi\left(u_{1}\right)\right) \in K$. Each element of $E(x) \cap B_{2}$ is mapped by $\pi$ to an element of $E(y) \cap B_{2}$. Moreover, each element of $E(y) \cap B_{2}$ must be the image under $\pi$ of an element of $E(x) \cap B_{2}$. We conclude that $\pi$ restricted to $E(x) \cap B_{2}$ is a bijection to $E(y) \cap B_{2}$, so $\left|E(x) \cap B_{2}\right|=\left|E(y) \cap B_{2}\right|$.

Given two term automata $A_{1}, A_{2}$, we define a partitioned graph $(U, E, P)$ :

$$
\begin{aligned}
U & =Q \cup\{\langle q, i\rangle \mid q \in Q \wedge \delta(q, i) \text { is defined }\} \\
E & =\{(q,\langle q, i\rangle) \mid \delta(q, i) \text { is defined }\} \\
& \cup\{(\langle q, i\rangle, \delta(q, i)) \mid \delta(q, i) \text { is defined }\} \\
L & =\left\{\left(q, q^{\prime}\right) \in Q \times Q \mid \ell(q)=\ell\left(q^{\prime}\right)\right\} \\
& \cup\left\{\left(\langle q, i\rangle,\left\langle q^{\prime}, i^{\prime}\right\rangle\right) \mid \ell(q)=\ell\left(q^{\prime}\right) \text { and if } \ell(q)=\rightarrow, \text { then } i=i^{\prime}\right\} \\
P & =U / L .
\end{aligned}
$$

The graph contains one node for each state and transition in $A_{1}, A_{2}$. Each transition in $A_{1}, A_{2}$ is mapped to two edges in the graph. This construction ensures that if a node in the graph corresponds to a state labeled $\prod^{n}$, then that node will have $n$ distinct successors in the graph. This is convenient when establishing a bijection between the successors of two nodes labeled $\prod^{n}$.

The equivalence relation $L$ creates a distinction between the two successors of a node that corresponds to a state labeled $\rightarrow$. This is done by ensuring that if $\left(\langle q, i\rangle,\left\langle q, i^{\prime}\right\rangle\right) \in L$ and $\ell(q)=\rightarrow$, then $i=i^{\prime}$. This is convenient when establishing a bijection between the successors of two nodes labeled $\rightarrow$.

Lemma 3. There exists a reflexive bisimulation $C$ between $A_{1}$ and $A_{2}$ such that $\left(q_{01}, q_{02}\right)$ $\in C$ if and only if there exists a size-stable refinement $S$ of $P$ such that $q_{01}$ and $q_{02}$ belong to the same block of $S$.

Proof. Let $C \subseteq Q \times Q$ be a reflexive bisimulation between $A_{1}$ and $A_{2}$ such that $\left(q_{01}, q_{02}\right) \in C$. Define an equivalence relation $K \subseteq U \times U$ such that:

$$
\begin{aligned}
K & =C \\
& \cup\left\{\left(\langle q, i\rangle,\left\langle q^{\prime}, i\right\rangle\right) \mid\left(q, q^{\prime}\right) \in C \wedge \ell(q)=\ell\left(q^{\prime}\right)=\rightarrow\right\} \\
& \cup\left\{\left(\langle q, i\rangle,\left\langle q^{\prime}, i^{\prime}\right\rangle\right) \mid\left(q, q^{\prime}\right) \in C \wedge\left(\delta(q, i), \delta\left(q^{\prime}, i^{\prime}\right)\right) \in C\right. \\
& \left.\wedge \ell(q)=\ell\left(q^{\prime}\right) \wedge \ell(q) \neq \rightarrow\right\} \\
S & =U / K .
\end{aligned}
$$

From $\left(q_{01}, q_{02}\right) \in C$, we have $\left(q_{01}, q_{02}\right) \in K$, so $q_{01}$ and $q_{02}$ belong to the same block of $S$. We will now show that $S$ is a size-stable refinement of $P$.

Let $\left(u, u^{\prime}\right) \in K$. From Lemma2 2 we have that it is sufficient to show that there exists a bijection $\pi: E(u) \rightarrow E\left(u^{\prime}\right)$, such that for all $u_{1} \in E(u)$, we have $\left(u_{1}, \pi\left(u_{1}\right)\right) \in K$. There are three cases.

First, suppose $\left(u, u^{\prime}\right) \in C$. We have

$$
\begin{aligned}
E(u) & =\{\langle u, i\rangle \mid \delta(u, i) \text { is defined }\} \\
E\left(u^{\prime}\right) & =\left\{\left\langle u^{\prime}, i^{\prime}\right\rangle \mid \delta\left(u^{\prime}, i^{\prime}\right) \text { is defined }\right\} .
\end{aligned}
$$


Let us consider each of the possible cases of $u$ and $u^{\prime}$. If $\ell(u)=\ell\left(u^{\prime}\right) \in \Gamma$, then $E(u)=E\left(u^{\prime}\right)=\emptyset$, and the desired bijection exists trivially. Next, if $\ell(u)=\ell\left(u^{\prime}\right)=\rightarrow$, then

$$
\begin{aligned}
E(u) & =\{\langle u, 0\rangle,\langle u, 1\rangle\} \\
E\left(u^{\prime}\right) & =\left\{\left\langle u^{\prime}, 0\right\rangle,\left\langle u^{\prime}, 1\right\rangle\right\},
\end{aligned}
$$

so the desired bijection is $\pi: E(u) \rightarrow E\left(u^{\prime}\right)$, where $\pi(\langle u, 0\rangle)=\left\langle u^{\prime}, 0\right\rangle$ and $\pi(\langle u, 1\rangle)=$ $\left\langle u^{\prime}, 1\right\rangle$, because $\left(\langle u, 0\rangle,\left\langle u^{\prime}, 0\right\rangle\right) \in K$ and $\left(\langle u, 1\rangle,\left\langle u^{\prime}, 1\right\rangle\right) \in K$. Finally, if $\ell(u)=$ $\ell\left(u^{\prime}\right)=\prod^{n}$, then

$$
\begin{aligned}
E(u) & =\{\langle u, i\rangle \mid \delta(u, i) \text { is defined }\} \\
E\left(u^{\prime}\right) & =\left\{\left\langle u^{\prime}, i^{\prime}\right\rangle \mid \delta\left(u^{\prime}, i^{\prime}\right) \text { is defined }\right\} .
\end{aligned}
$$

From $\left(u, u^{\prime}\right) \in C$, we have a bijection $b:\{0 . . n-1\} \rightarrow\{0 . . n-1\}$ such that $\forall i \in\{0 . . n-$ $1\}:\left(\delta(u, i), \delta\left(u^{\prime}, b(i)\right)\right) \in C$. From that, the desired bijection can be constructed.

Second, suppose $u=\langle q, i\rangle$ and $u^{\prime}=\left\langle q^{\prime}, i\right\rangle$, where $\left(q, q^{\prime}\right) \in C$, and $\ell(q)=$ $\ell\left(q^{\prime}\right)=\rightarrow$. We have

$$
\begin{aligned}
E(u) & =\{\delta(q, i)\} \\
E\left(u^{\prime}\right) & =\left\{\delta\left(q^{\prime}, i\right)\right\},
\end{aligned}
$$

and from $\left(q, q^{\prime}\right) \in C$ we have $\left(\delta(q, i), \delta\left(q^{\prime}, i\right)\right) \in C \subseteq K$, so the desired bijection exists.

Third, suppose $u=\langle q, i\rangle$ and $u^{\prime}=\left\langle q^{\prime}, i^{\prime}\right\rangle$, where $\left(q, q^{\prime}\right) \in C,\left(\delta(q, i), \delta\left(q^{\prime}, i^{\prime}\right)\right) \in$ $C, \ell(q)=\ell\left(q^{\prime}\right)$, and $\ell(q) \neq \rightarrow$. We have

$$
\begin{aligned}
E(u) & =\{\delta(q, i)\} \\
E\left(u^{\prime}\right) & =\left\{\delta\left(q^{\prime}, i^{\prime}\right)\right\},
\end{aligned}
$$

and $\left(\delta(q, i), \delta\left(q^{\prime}, i^{\prime}\right)\right) \in C \subseteq K$, so the desired bijection exists.

Conversely, let $S$ be a size-stable refinement of $P$ such that $q_{01}$ and $q_{02}$ belong to the same block of $S$. Define:

$$
\begin{aligned}
& K=\left\{\left(u, u^{\prime}\right) \in U \times U \mid u, u^{\prime} \text { belong to the same block of } S\right\} \\
& C=K \cap(Q \times Q) .
\end{aligned}
$$

Notice that $\left(q_{01}, q_{02}\right) \in C$ and that $C$ is reflexive. We will now show that $C$ is a bisimulation between $A$ and $A^{\prime}$.

First, suppose $\left(q, q^{\prime}\right) \in C$. From $S$ being a refinement of $P$ we have $\left(q, q^{\prime}\right) \in L$, so $\ell(q)=\ell\left(q^{\prime}\right)$.

Second, suppose $\left(q, q^{\prime}\right) \in C$ and $\ell(q)=\longrightarrow$. From the definition of $E$ we have

$$
\begin{aligned}
E(q) & =\{\langle q, 0\rangle,\langle q, 1\rangle\} \\
E\left(q^{\prime}\right) & =\left\{\left\langle q^{\prime}, 0\right\rangle,\left\langle q^{\prime}, 1\right\rangle\right\} .
\end{aligned}
$$


From $S$ being size-stable, $\left(q, q^{\prime}\right) \in C \subseteq K$, and Lemma 2 we have that there exists a bijection $\pi: E(q) \rightarrow E\left(q^{\prime}\right)$ such that for all $u \in E(q)$ we have that $(u, \pi(u)) \in K$. From $K \subseteq L$ and $\ell(q)=\longrightarrow$ we have that there is only one possible bijection $\pi$ :

$$
\begin{aligned}
& \pi(\langle q, 0\rangle)=\left\langle q^{\prime}, 0\right\rangle \\
& \pi(\langle q, 1\rangle)=\left\langle q^{\prime}, 1\right\rangle,
\end{aligned}
$$

so $\left(\langle q, 0\rangle,\left\langle q^{\prime}, 0\right\rangle\right) \in K$ and $\left(\langle q, 1\rangle,\left\langle q^{\prime}, 1\right\rangle\right) \in K$. From the definition of $E$ we have, for $i \in\{0,1\}$,

$$
\begin{gathered}
E(\langle q, i\rangle)=\delta(q, i) \\
E\left(\left\langle q^{\prime}, i\right\rangle\right)=\delta\left(q^{\prime}, i\right),
\end{gathered}
$$

and since $S$ is size-stable, we have, for $i \in\{0,1\},\left(\delta(q, i), \delta\left(q^{\prime}, i\right)\right) \in K$. Moreover, for $i \in\{0,1\},\left(\delta(q, i), \delta\left(q^{\prime}, i\right)\right) \in Q \times Q$, so we conclude, $\left(\delta(q, i), \delta\left(q^{\prime}, i\right)\right) \in C$.

Third, suppose $\left(q, q^{\prime}\right) \in C$ and $\ell(q)=\prod^{n}$. From the definition of $E$ we have

$$
\begin{aligned}
E(q) & =\{\langle q, i\rangle \mid \delta(q, i) \text { is defined }\} \\
E\left(q^{\prime}\right) & =\left\{\left\langle q^{\prime}, i\right\rangle \mid \delta\left(q^{\prime}, i\right) \text { is defined }\right\} .
\end{aligned}
$$

Notice that $|E(q)|=\left|E\left(q^{\prime}\right)\right|=n$. From $S$ being size-stable, $\left(q, q^{\prime}\right) \in C \subseteq K$, and Lemma 2 we have that there exists a bijection $\pi: E(q) \rightarrow E\left(q^{\prime}\right)$ such that for all $u \in E(q)$ we have that $(u, \pi(u)) \in K$. From $\pi$ we can derive a bijection $b:\{0 . . n-1\} \rightarrow\{0 . . n-1\}$ such that $\forall i \in\{0 . . n-1\}:\left(\langle q, i\rangle,\left\langle q^{\prime}, b(i)\right\rangle\right) \in K$. From the definitions of $E$ and $E^{\prime}$ we have that for $i \in\{0 . . n-1\}$,

$$
\begin{aligned}
E(\langle q, i\rangle) & =\{\delta(q, i)\} \\
E\left(\left\langle q^{\prime}, i\right\rangle\right) & =\left\{\delta\left(q^{\prime}, i\right)\right\},
\end{aligned}
$$

and since $S$ is size-stable, and, for all $i \in\{0 . . n-1\},\left(\langle q, i\rangle,\left\langle q^{\prime}, b(i)\right\rangle\right) \in K$, we have $\left(\delta(q, i), \delta\left(q^{\prime}, b(i)\right)\right) \in K$. Moreover, $\left(\delta(q, i), \delta\left(q^{\prime}, b(i)\right)\right) \in Q \times Q$, so we conclude $\left(\delta(q, i), \delta\left(q^{\prime}, b(i)\right)\right) \in C$.

The size of a term automata $A=\left(Q, \Sigma, q_{0}, \delta, l\right)$ is $|Q|+|| \delta \mid$, i.e., the sum of the number of states and transitions in the automata.

Theorem 1. For types $\tau_{1}, \tau_{2}$ that can be represented by term automata $A_{1}, A_{2}$ of size at most $n$, we can decide $\left(\tau_{1}, \tau_{2}\right) \in \mathcal{R}$ in $O(n \log n)$ time.

Proof. From Lemma 1 we have that $\left(\tau_{1}, \tau_{2}\right) \in \mathcal{R}$ if and only if there is a reflexive bisimulation $C$ between $A_{1}$ and $A_{2}$ such that $\left(q_{01}, q_{02}\right) \in C$. From Lemma 3 we have that there exists a reflexive bisimulation $C$ between $A_{1}$ and $A_{2}$ such that $\left(q_{01}, q_{02}\right) \in C$ if and only if there exists a size-stable refinement $S$ of $P$ such that $q_{01}$ and $q_{02}$ belong to the same block of $S$.

Paige and Tarjan [16] give an $O(m \log p)$ algorithm to find the coarsest size-stable refinement of $P$, where $m$ is the size of $E$ and $p$ is the size of the universe $U$.

Our algorithm first constructs $(U, E, P)$ from $A_{1}$ and $A_{2}$, then runs the Paige-Tarjan algorithm to find the coarsest size-stable refinement $S$ of $P$, and finally checks whether $q_{01}$ and $q_{02}$ belong to the same block of $S$. 
If $A_{1}$ and $A_{2}$ are of size at most $n$, then the size of $E$ is at most $2 n$, and the size of $U$ is at most $2 n$, so the total running time of our algorithm is $O(2 n \log (2 n))=O(n \log n)$.

Next, we illustrate how our algorithm determines that equivalence between the types. Details of the algorithm can be found in [16]. Consider two types $I_{1}$ and $J_{1}$ defined in Section 2 The set of types corresponding to the two interfaces are:

$$
\begin{aligned}
& \left\{I_{1}, I_{2}, m_{1}, m_{2}, m_{3}, m_{4}, \text { int }, \text { float }\right\} \\
& \left\{J_{1}, J_{2}, n_{1}, n_{2}, n_{3}, n_{4}, \text { int }, \text { float }\right\}
\end{aligned}
$$

Figure 5 shows various steps of our algorithm. For simplicity, the figure only shows the blocks of actual types, but not the blocks of the extra nodes of the form $\langle q, i\rangle$. The blocks in the first row are based on labels, e.g., states labeled with $\times$ are in the same block. In the next step, the block containing the methods are split based on the type of the result of the method, e.g.. methods $m_{1}$ and $n_{4}$ both return float, so they are in the same block. In the next step (corresponding to the third row) the block $\left\{I_{1}, I_{2}, J_{1}, J_{2}\right\}$ are split. The final partition, where block $\left\{m_{3}, m_{4}, n_{1}, n_{2}\right\}$ is split, is shown in the fourth row.

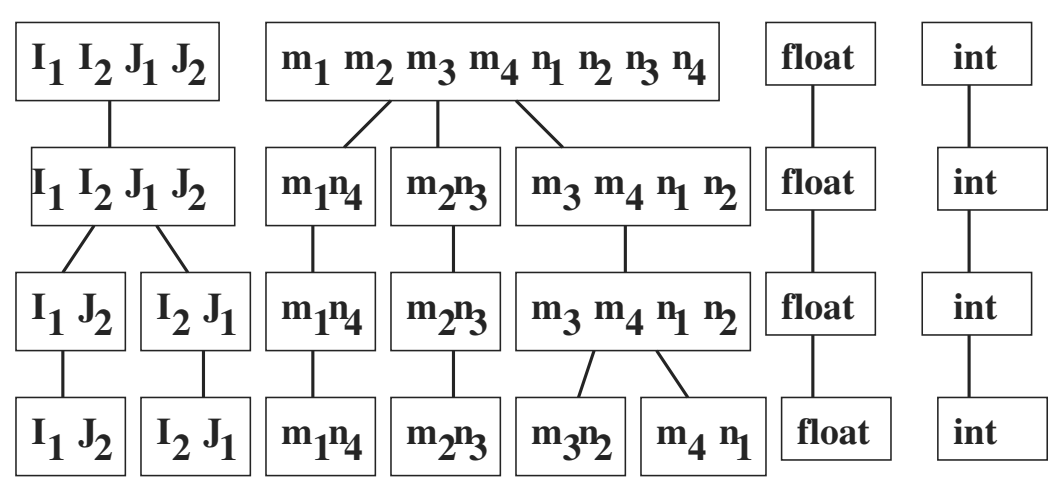

Fig. 5. Blocks of types

Our algorithm can be tuned to take a specific user needs into account. This is done simply by modifying the definition of the equivalence relation $L$. For example, suppose a user cares about the order of the arguments to a method. This means that the components of the product type that models the argument list should not be allowed to be shuffled during type matching. We can prevent shuffling by employing the same technique that the current definition of $L$ uses for function types. The idea is to insist that two component types may only be matched when they have the same component index.

Another example of the tunability of our algorithm involves the modifiers in Java. Suppose a programmer is developing a product that is multi-threaded. In this case the programmer may only want to match synchronized methods with other synchronized methods. This can be handled easily in our framework by changing $L$ such that two method types may only be matched when they are both synchronized. On the other 
hand if the user is working on a single-threaded product, the keyword synchronized can be ignored. The same observation applies to other modifiers such as static.

\section{Our Implementation}

We have implemented our algorithm in Java and the current version is based on the code written by Wanjun Wang. The implementation and documentation are freely available at

http://www.cs.purdue.edu/homes/tzhao/matching/matching.htm.

The current version has a graphical user interface so that users may input type definitions written in a file and also may specify restrictions on type isomorphism.

Suppose we are given the following file with four Java interfaces.

$$
\begin{aligned}
& \text { interface } I_{1}\left\{\quad \text { interface } I_{2}\{\right. \\
& \text { float } \left.m_{1}\left(I_{1} \text { a, int } b\right) ; \quad J_{2} m_{3} \text { (float } a\right) \text {; } \\
& \text { \} } \\
& \text { int } m_{2} \quad\left(I_{2} a\right) \text {; } \\
& \text { interface } J_{1}\{ \\
& \left.I_{1} n_{1} \text { (float } a\right) ; \quad \text { int } n_{3}\left(J_{1} a\right) \text {; } \\
& \text { \} }
\end{aligned}
$$

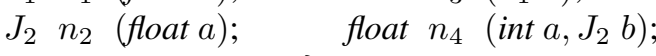

The implementation, as illustrated in the Figure 6, will read and parse the input file and then transform the type definitions into partitions of numbers with each type definition and dummy type assigned a unique number. The partitions will be refined by Paige-Tarjan algorithm until it is size-stable as defined in this paper. Finally, we will be

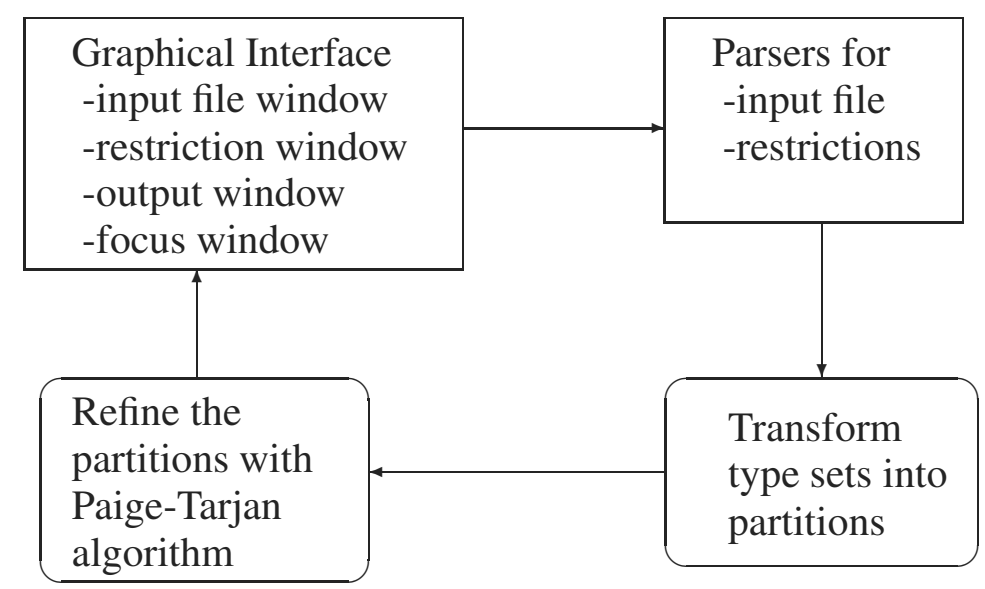

Fig. 6. Schematic diagram for the implementation 
able to read the results from the final partitions. Two types are isomorphic if the numbers assigned to them are in the same partition.

The implementation will give the following output:

$$
\begin{aligned}
I_{1} & =J_{2} \\
I_{2} & =J_{1} \\
I_{1} \cdot m_{1} & =J_{2} \cdot n_{4} \\
I_{2} \cdot m_{3} & =I_{2} \cdot m_{4}=J_{1} \cdot n_{1}=J_{1} \cdot n_{2} \\
I_{1} \cdot m_{2} & =J_{2} \cdot n_{3} \cdot
\end{aligned}
$$

We can see that the types of interfaces $I_{2}$ and $J_{1}$ are isomorphic and moreover, all method types of $I_{2}, J_{1}$ match. Suppose that we have additional information about the method types such that only method $m_{3}$ and $n_{1}$ should have isomorphic types. We can restrict the type matching by adding $I_{2} \cdot m_{3}=J_{1} \cdot n_{1}$ to the restrictions window of the user interface. The new matching result is illustrated by the screen shot in figure 7 .

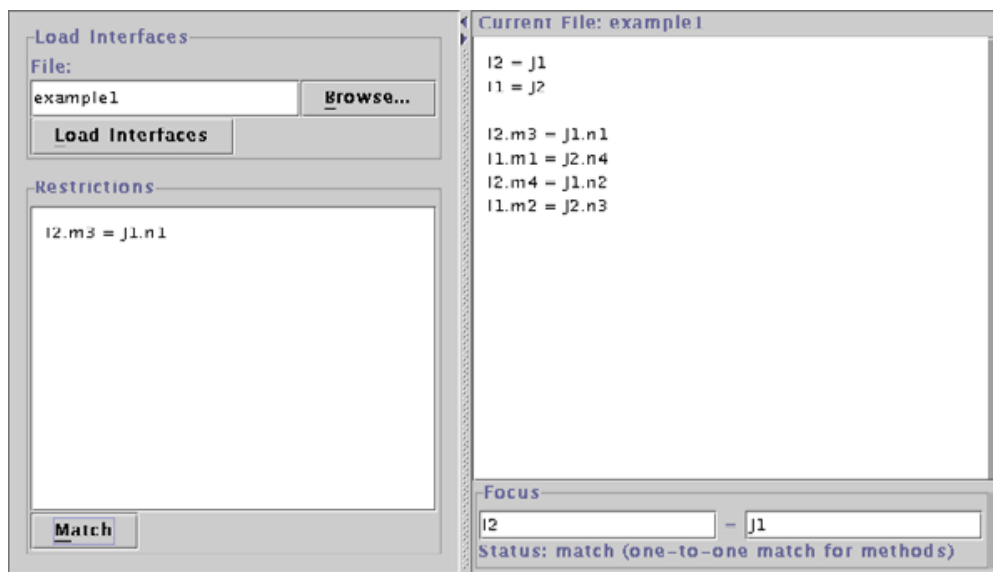

Fig. 7. Screen shot

Note that we are able to focus on the matching of two interface types such as $I_{2}, J_{1}$ as in the focus windows of Figure 7, where $I_{2}, J_{1}$ are matched and their methods are matched one to one.

\section{Subtyping of Recursive Types}

In this section we discuss subtyping and formalize it using a simulation relation. We also discuss reasons why the algorithm given in Section 4 is not applicable to subtyping of of recursive types. Consider the interfaces $I_{1}$ and $I_{2}$ shown in Figure 8 , and suppose a 
Let $A_{1}, A_{2}$ denote two term automata over $\Sigma$ :

$$
\begin{aligned}
& A_{1}=\left(Q_{1}, \Sigma, q_{01}, \delta_{1}, \ell_{1}\right) \\
& A_{2}=\left(Q_{2}, \Sigma, q_{02}, \delta_{2}, \ell_{2}\right) .
\end{aligned}
$$

We assume that $Q_{1} \cap Q_{2}=\emptyset$. Define $Q=Q_{1} \cup Q_{2}, \delta: Q \times \omega \rightarrow Q$ where $\delta=\delta_{1} \oplus \delta_{2}$, and $\ell: Q \rightarrow \Sigma$, where $\ell=\ell_{1} \oplus \ell_{2}$, where $\oplus$ denotes disjoint union of two functions. We say that $A_{2}$ simulates $A_{1}$ (denoted by $A_{1} \preceq A_{2}$ ) if and only if there exists a relation $D \subseteq Q \times Q$, called a simulation relation between $A_{1}$ and $A_{2}$, such that:

- if $\left(q, q^{\prime}\right) \in D$ and $\ell(q) \in \Gamma$, then $\ell\left(q^{\prime}\right) \in \Gamma$ and $\left.\ell(q) \preceq \ell\left(q^{\prime}\right)\right)$.

- if $\left(q, q^{\prime}\right) \in D$ and $\ell(q) \in\left(\{\rightarrow\} \cup\left\{\prod^{n}, n \geq 2\right\}\right)$, then $\ell(q)=\ell\left(q^{\prime}\right)$.

- if $\left(q, q^{\prime}\right), \in D$ and $\ell(q)=\longrightarrow$, then $\left(\delta(q, 0), \bar{\delta}\left(q^{\prime}, 0\right)\right) \in D$ and $\left(\delta(q, 1), \delta\left(q^{\prime}, 1\right) \in D\right.$.

- if $\left(q, q^{\prime}\right), \in D$ and $\ell(q)=\prod^{n}$, then there exists a bijection $b:\{0 \cdots n-1\} \rightarrow$ $\{0 \cdots n-1\}$ such that for all $i \in\{0 \cdots n-1\}$, we have $\left(\delta(q, i), \delta\left(q^{\prime} i\right)\right) \in D$.

- Suppose $\left(q, q^{\prime}\right) \in D$ and $\ell(q)=x^{n}$. If $\ell\left(q^{\prime}\right) \notin\left\{x^{m}, m \geq 2\right\}$, then there exists a $j \in\{0 \cdots n-1\}$ such that $\left(\delta(q, j), q^{\prime}\right) \in D$. Otherwise, assume that $\ell\left(q^{\prime}\right)=\times^{m}$, where $m \leq n$ and in this case, there exists an injective function $c:\{0 \cdots m-1\} \rightarrow$ $\{0 \cdots n-1\}$ such that for all $i \in\{0 \cdots m-1\}$, we have $\left(\delta(q, c(i)), \delta\left(q^{\prime}, i\right)\right) \in D$.

Notice that the simulations between $A_{1}$ and $A_{2}$ are closed under union, therefore, there exists a largest simulation between $A_{1}$ and $A_{2}$. The proof of Lemma 4 is similar to the proof of Lemma 1 and is omitted.

Lemma 4. For types $\tau_{1}, \tau_{2}$ that are represented by the term automata $A_{1}, A_{2}$, respectively, we have $\left(\tau_{1}, \tau_{2}\right) \in \mathcal{S}$ if and if only there is a reflexive simulation $D$ between $A_{1}$ and $A_{2}$ such that $\left(q_{01}, q_{02}\right) \in D$.

The largest simulation between the term automata $A_{1}$ and $A_{2}$ is given by the following greatest fixed point

$$
\nu D \cdot \operatorname{sim}\left(q, q^{\prime}, D\right) \text {. }
$$

where $D \subseteq Q_{1} \times Q_{2}$ and the predicate $\operatorname{sim}\left(q, q^{\prime}, D\right)$ is the conjunction of the five conditions which appear in the definition of the simulation relation between two automata. Let $n$ and $m$ be the size of the term automata $A_{1}$ and $A_{2}$ respectively. Since $n m$ is a bound on the size of $D$, the number of iterations in computing the greatest fixed point is bounded by $n m$. In general, the relation $D$ (or for that matter the simulation relation) is not symmetric. On the other hand, the bisimulation relation was an equivalence relation, and so could be represented as a partition on the set $Q_{1} \cup Q_{2}$, or in other words, partitions give us a representation of an equivalence relation that is linear in the sum of the sizes of the set of states $Q_{1}$ and $Q_{2}$. The Paige-Tarjan algorithm uses the partition representation of the equivalence relation. Since $D$ is not symmetric (and thus not an equivalence relation), it cannot be represented by a partition. This is the crucial reason why our previous algorithm cannot be used for subtyping.

\section{Conclusion}

In this paper we addressed the problem of matching recursive types. We presented an algorithm with $O(n \log n)$ time complexity that determines whether two types are 
equivalent. To our knowledge, this is the most efficient algorithm for this problem. Our results are applicable to the problem of matching signatures of software components. Applications to Java were also discussed. Issues related to subtyping of recursive types were also addressed. Next we discuss potential applications of our algorithm.

CORBA: The CORBA approach utilizes a separate definition language called IDL. Objects are associated with language-independent interfaces defined in IDL. These interfaces are then translated into the language being used by the client. The translated interface then enables the clients to call the objects. Since the IDL interfaces have to be translated into several languages, their type system is very restrictive. Therefore, IDL interfaces lack expressive power because, intuitively speaking, the type system used in IDL has to be the intersection of the type system for each language it supports. The drawback of for CORBA-style approaches to interoperability are well articulated in [34].

Polyspin and Mockingbird: The Polyspin and Mockingbird approaches do not require a common interface language, such as IDL. In both these approaches, clients and objects are written in languages with separate type systems, and an operation that crosses the language boundary is supported by bridge code that is automatically generated. Therefore, systems such as Polyspin and Mockingbird support seamless interoperability since the programmer is not burdened with writing interfaces in a second language, such as IDL in CORBA. Polyspin supports only finite types. Mockingbird on the other hand supports recursive types, including records, linked lists, and arrays. The type system used in Mockingbird is called the Mockingbird Signature Language or MockSL. The problem of deciding type equivalence for MockSL remains open [2]. In this paper we considered a type system which is related to the one used in Mockingbird. However, we are investigating a translation from MockSL to recursive types.

Megaprogramming: Techniques suitable for very large software systems have been a major goal of software engineering. The term megaprogramming was introduced by DARPA to motivate this goal [5]. Roughly speaking, in megaprogramming, megamodules provide a higher level of abstraction than modules or components. For example, a megamodule can encapsulate the entire logistics of ground transportation in a major city. Megaprogramming is explained in detail in [22]. Interoperability issues arise when megaprograms are constructed using megamodules (see [22, Section 4.2]). We believe that the framework presented in this paper can be used to address mismatch between interfaces of megamodules.

Future work includes investigating type inference. Coppo's recent paper [9] on type inference with recursive type equations may contain techniques that are applicable.

\section{References}

1. Roberto M. Amadio and Luca Cardelli. Subtyping recursive types. ACM Transactions on Programming Languages and Systems, 15(4):575-631, 1993. Also in Proceedings of POPL'91.

2. Joshua Auerbach, Charles Barton, and Mukund Raghavachari. Type isomorphisms with recursive types. Research report RC 21247, IBM Research Division, T. J. Watson Research Center, Yorktown Heights, NY, August 1998.

3. Joshua Auerbach and Mark C. Chu-Carroll. The mockingbird system: A compiler-based approach to maximally interoperable distributed programming. Research report RC 20718, 
IBM Research Division, T. J. Watson Research Center, Yorktown Heights, NY, February 1997.

4. Daniel J. Barrett, Alan Kaplan, and Jack C. Wileden. Automated support for seamless interoperability in polylingual software systems. In ACM SIGSOFT'96, Fourth Symposium on the Foundations of Software Engineering, San Francisco, California, October 1996.

5. B. Boehm and B. Scherlis. Megaprogramming. In Proceedings of DARPA Software Technology Conference, April 28-30, Meridien Corporation, Arlington, VA 1992.

6. Michael Brandt and Fritz Henglein. Coinductive axiomatization of recursive type equality and subtyping. In Proceedings of TLCA'97, 3rd International Conference on Typed Lambda Calculus and Applications, 1997.

7. Kim B. Bruce, Roberto Di Cosmo, and Giuseppe Longo. Provable isomorphisms of types. Mathematical Structures in Computer Science, 2(2):231-247, 1992.

8. Luca Cardelli and Peter Wegner. On understanding types, data abstraction, and polymorphism. ACM Computing Surveys, 17(4):471-522, December 1985.

9. Mario Coppo. Type inference with recursive type equations. In Proceedings of FOSSACS'01, Foundations of Software Science and Computation Structures, pages 184-198. SpringerVerlag (LNCS 2030), 2001.

10. Roberto Di Cosmo. Isomorphisms of Types: from $\lambda$-calculus to information retrieval and language design. Birkhäuser, 1995.

11. Bruno Courcelle. Fundamental properties of infinite trees. Theoretical Computer Science, 25(1):95-169, 1983.

12. Trevor Jim and Jens Palsberg. Type inference in systems of recursive types with subtyping. Manuscript, 1997.

13. Dexter Kozen, Jens Palsberg, and Michael I. Schwartzbach. Efficient recursive subtyping. Mathematical Structures in Computer Science, 5(1):113-125, 1995. Preliminary version in Proceedings of POPL'93, Twentieth Annual SIGPLAN-SIGACT Symposium on Principles of Programming Languages, pages 419-428, Charleston, South Carolina, January 1993.

14. Paliath Narendran, Frank Pfenning, and Richard Statman. On the unification problem for Cartesian closed categories. In Proceedings, Eighth Annual IEEE Symposium on Logic in Computer Science, pages 57-63. IEEE Computer Society Press, 1993.

15. OMG. The common object request broker: Architecture and specification. Technical report, Object Management Group, 1999. Version 2.3.1.

16. Robert Paige and Robert Tarjan. Three partition refinement algorithms. SIAM Journal on Computing, 16(6):973-989, December 1987.

17. Jens Palsberg and Tian Zhao. Efficient and flexible matching of recursive types. Information and Computation, to appear. Preliminary version in Proceedings of LICS'00, Fifteenth Annual IEEE Symposium on Logic in Computer Science, pages 388-398, Santa Barbara, California, June 2000.

18. Mikael Rittri. Retrieving library identifiers via equational matching of types. In M. E. Stickel, editor, Proceedings of the 10th International Conference on Automated Deduction, volume 449 of LNAI, pages 603-617, Kaiserslautern, FRG, July 1990. Springer Verlag.

19. Mikael Rittri. Using types as search keys in function libraries. Journal of Functional Programming, 1(1):71-89, 1991.

20. Mikael Rittri. Retrieving library functions by unifying types modulo linear isomorphism. RAIRO Theoretical Informatics and Applications, 27(6):523-540, 1993.

21. Sergei V. Soloviev. The category of finite sets and cartesian closed categories. Journal of Soviet Mathematics, 22:1387-1400, 1983.

22. G. Wiederhold, P. Wegner, and S. Ceri. Towards Megaprogramming: A paradigm for component-based programming. Communications of the ACM, 35(11):89-99, November 1992. 
23. A. M. Zaremski and J. M. Wing. Signature matching: a tool for using software libraries. ACM Transactions on Software Engineering Methodology, 4(2):146-170, April 1995.

24. A. M. Zaremski and J. M. Wing. Specification matching of software components. In Proceedings of 3rd ACM SIGSOFT Symposium on the Foundation of Software Engineering, pages 6-17, 1995. 